\title{
Collective Creativity: The Emergence of World of Warcraft Machinima
}

\author{
Tyler Pace \\ Indiana University \\ School of Informatics and Computing \\ $901 \mathrm{E} 10^{\text {th }} \mathrm{St}$ \\ Bloomington IN, 47408 USA \\ tympace@indiana.edu
}

\author{
Jeffrey Bardzell \\ Indiana University \\ School of Informatics and Computing \\ $919 \mathrm{E} 10^{\text {th }} \mathrm{St}$ \\ Bloomington IN, 47408 USA \\ jbardzel@indiana.edu
}

\author{
Shaowen Bardzell \\ Indiana University \\ School of Informatics and Computing \\ $919 \mathrm{E} 10^{\text {th }} \mathrm{St}$ \\ Bloomington IN, 47408 USA \\ selu@indiana.edu
}

\begin{abstract}
$\mathrm{HCls}$ interest in creativity support has extended beyond the realm of information-oriented professionals to the efforts of experience-driven collective communities. World of Warcraft machinima provides an opportunity to study networked creativity among a widely distributed and highly productive amateur community. We present an analysis of metadata gathered from the most viewed machinima on YouTube and WarcraftMovies. We demonstrate (1) a means for selecting an accessible corpus from a large population of videos, and (2) provide early evidence to support the claim that collective creativity develops over time and reaches a point of stabilization of production practices, technological infrastructure, aesthetic forms, and critical appreciation.
\end{abstract}

\section{Creativity, Network, Online, Machinima, Internet Video, World of Warcraft, YouTube}

\section{INTRODUCTION}

Internet-based special interest communities have demonstrated enormous creativity and productivity in recent years, from Wikipedia and open source communities to art, craft, and multimedia communities, such as Second Life, Deviant Art, and Etsy.com, to name a few. Increasingly, such communities are the subjects of $\mathrm{HCl}$ research, as scholars seek to understand the organizational structures and productive processes that support these often bottom-up, non-traditional collaborations, such as knowledge management and quality control (Kriplean et al., 2007). Other work has focused on community formation and maintenance (Kow and Nardi, 2010; Pearce, 2009). Such research can influence the design of technologies intended to support community-level creative collaboration in both amateur (Bardzell, 2007) and professional communities (Aragon et al., 2009).

Much of this work, however, emphasizes the management and production of information; alternatively, we can consider creative participation in such communities in terms of experience. For while the production of information and knowledge is obviously central to communities such as Wikipedia, the incentive to have a certain kind of experience is also critical, especially so to entertainment, multimedia, and art- and craft-related communities. We follow (Dewey, 1934; McCarthy and Wright, 2004) in believing that creativity is frequently a quality or feature of a good experience. Thus, investigating the relationship between creativity and technology can simultaneously contribute to research on creativity-support technologies and good user experiences.

The present work focuses on the community surrounding World of Warcraft (WoW) machinima. World of Warcraft is a massively multiplayer online roleplaying game, with an estimated 12 million paying subscribers. Machinima refers to digital videos that are produced (i.e., set, acted out, and recorded) in video games. Most of these videos are 3-5 minutes in length, and in addition to showing actual, staged, and/or composited game play may also feature any combination of in-game sounds, original voice acting, original music, and recordings taken from popular culture (e.g., pop songs and movies). The WoW machinima community includes the video producers themselves, their audiences, and those who develop tools for them. In addition to studying the structure of the community and common production practices, we also seek to develop a historical understanding of the machinima videos themselves.

At this stage, we have two research objectives: (1) to identify a corpus appropriate to study and (2) to test the hypothesis that production techniques, content creation tools, and community standards coevolved and achieved a point of stability or critical mass. We begin by motivating (1). With tens of 
thousands of WoW machinima videos available online, we need to find a way to identify which subset of videos is most appropriate for study. One way is to sample randomly, a technique that could identify general content trends. However, our goal is not to sample the whole field, but rather to identify the most important videos. Doing so would provide us with the benefits of a canon, akin to the literary canon or the cinematic canon, i.e., an ability to trace the relationships among the most important videos over time, in terms of production techniques, semantic contents, and audience reception.

With even an approximation of a canon, we could be in a position to consider (2). We know from media studies and film history that new media forms are often made possible due to innovations in technology and also that it takes time for a community to learn how to use that technology to create stable aesthetic genres. For example, film historians note that whereas film became technologically possible in the 1890s, what we recognize today as modern cinema (e.g., a certain combination of shot composition, acting technique, editing, and storytelling) only came together around 1930. Of course, innovations in both technology and cinematic expression continue through to the present, but the first 40 years mark an especially dynamic period where the cinematic aesthetic was discovered and developed.

Thus, it is reasonable to suppose that WoW machinima similarly goes through a developmental period, in which the community collectively figures out how to produce, evaluate, and disseminate "good" (according to the community) WoW machinima. At some critical point according to this hypothesis, WoW machinima would stabilize as a genre, and future innovation would happen more incrementally thenceforth. The emergence itself of a new genre, both in terms of production techniques and a critical aesthetic, is a significant creative achievement in its own right, and it is this kind of achievement we hope to understand as an important outcome of certain online communities. When we seek to support the creativity of collectives, therefore, the evolution and stabilization of genres should be among the forms of creativity that we target.

\section{METHODOLOGY}

This paper reports a quantitative analysis on audience metrics of WoW machinima over time. This analysis not only helps us develop an approximation of a canon (we narrow the field to 205 top videos down from over 10,000), but it also provides early evidence that there is a watershed year, in which WoW machinima stabilizes as an aesthetic genre for its community: 2007.
One of the benefits of analysing Internet born amateur multimedia is the extensive metadata that is automatically generated alongside the media. For Internet video, metadata frequently includes the number of views, ratings, date posted, incoming referrals, uploader description, viewer comments, etc. We used such data to construct a corpus of videos to serve as a rough approximation of our final goal: the canon of the most important WoW machinima. Such a metadata-derived corpus lends itself to quantifiable/statistical analysis, since collected videos are already associated with rich historical data. However, a key limitation to this approach, to whose implications we'll return later in the paper, is the fact that such a corpus lacks any curation, i.e., confirmation from community experts that this corpus approximates the canon well.

The corpus developed for this project is based on videos uploaded to the two primary locations for World of Warcraft machinima: YouTube.com and WarcraftMovies.com. While many more sites were included in our original search for WoW machinima, it quickly became apparent that both YouTube and Warcraft Movies were the major sources of WoW machinima in terms of the total number of machinima posted to the site. YouTube hosts at least 7,500 WoW machinima-tagged videos (limitations in the YouTube search prevent a more precise measure) and Warcraft Movies hosts 3,746 machinima at the time of this writing.

Because YouTube.com is the major repository of WoW machinima, it was used as the "test" location to develop the metadata criteria for which machinima to include in the corpus. The number of views per video was selected as the primary criterion for inclusion because the number of views is both resistant to grouping (i.e., videos are less likely to have the same number of views as they are the same rating) and helps to incorporate a populist view of machinima (i.e., highly watched videos are likely watched for a reason, good or bad). A scraper recorded the view count from a significant sample of WoW machinima labelled videos on YouTube.com. In that sample, the top 5\% (a conservative measure of the top percentile) of videos averaged 200,000 or more views each. As a result, 200,000 views became the cut off point for inclusion in the corpus.

Applying the criteria of 200,000 or more views to the population of WoW machinima labelled films on YouTube.com produced 190 films for inclusion in the corpus. The same metric applied to WarcraftMovies.com yielded only 15 videos for inclusion into the corpus for a total of 205 videos. More details about the corpus and a breakdown of the videos per location are available in Table 1. 
Table 1: Breakdown of Machinima Corpus by Video Location

\begin{tabular}{|r|c|c|c|c|c|}
\hline & $\begin{array}{l}\text { Total Est. } \\
\text { Machinima }\end{array}$ & $\begin{array}{l}\text { Number in } \\
\text { Corpus }\end{array}$ & Total Views & $\begin{array}{l}\text { Avg. Views per } \\
\text { Machinima }\end{array}$ & $\begin{array}{l}\text { Avg. Rating per } \\
\text { Machinima (\%) }\end{array}$ \\
\hline YouTube.com & $>7,500$ & 190 & $322,043,543$ & $1,694,966$ & 91.03 \\
\hline WarcraftMovies.com & 3,746 & 15 & $9,339,067$ & 622,604 & 96.77 \\
\hline Totals & - & 205 & $331,382,610$ & $1,158,785$ (Avg.) & 93.90 (Avg.) \\
\hline
\end{tabular}

For each video in the corpus, the following metadata was collected: date posted, number of views, rating, uploader username, video description, and author/producer name when possible. Rating scales from YouTube.com and WarcraftMovies.com were normalized to a $0-100 \%$ scale for comparison across the two sites. Upload dates were adjusted for videos when the description noted that the video was a copy (machinima is sometimes removed for copyright violations and reuploaded at a later date). Author and uploader names were matched when information in the video description or the video itself identified the author and uploader as the same individual or group. Finally, the adjusted metadata for the WoW machinima corpus was analysed for significant relations. Our primary interest is in identifying trends among the number of authors/producers, number of videos, views, and ratings over time. Results from the analysis are presented in the following section.

\section{RESULTS}

In the following section, we present summary data about authors, videos, views and ratings for the collected machinima corpus. This data is then used to develop a number of models that illustrate significant changes in the number of videos, number of views, and video ratings over time. These individual models are then combined into a unified model that demonstrates an inverse relation between video views and ratings over time for WoW machinima.

Table 2: Authors and Videos, Views and Ratings

\begin{tabular}{|l|c|c|c|}
\hline $\begin{array}{l}\text { Authors } \\
(\mathbf{n}=99)\end{array}$ & $\begin{array}{c}\text { Num. } \\
\text { Videos }\end{array}$ & $\begin{array}{c}\text { Num. } \\
\text { Views }\end{array}$ & $\begin{array}{c}\text { Avg. } \\
\text { Ratings (\%) }\end{array}$ \\
\hline Mean & 2 & $3,403,798$ & 90.07 \\
\hline Median & 1 & 947,878 & 94.03 \\
\hline Std. Dev. & 5 & $7,409,110$ & 12.67 \\
\hline Range & 46 & $44,338,973$ & 85.63 \\
\hline
\end{tabular}

Table 3: Summary for Top Machinima Author "Oxhorn" Values marked with * are significant at $p<.01$.

\begin{tabular}{|l|c|c|}
\hline Videos (n=46) & Views & $\begin{array}{c}\text { Ratings } \\
(\%)\end{array}$ \\
\hline Mean & ${ }^{*} 995,080$ & ${ }^{*} 94.97$ \\
\hline Median & ${ }^{\star} 537,602$ & ${ }^{*} 95.62$ \\
\hline Std. Dev. & $1,874,654$ & 2.69 \\
\hline Range & $12,390,419$ & 13.57 \\
\hline Total & $43,783,521$ & -- \\
\hline & &
\end{tabular}

Table 2 presents a breakdown of data for all authors in the WoW machinima corpus. While the exact number of authors is unknown (authors might change their usernames over time), there are 99 unique author usernames in the corpus. These potential authors have an average of 1 video each with a range of 46 videos. Further, the average number of total views for each author is approximately $3.4 \mathrm{~m}$ with a range of total views of $44 \mathrm{~m}$. In other words, views are not evenly spread among authors with a few key authors obtaining a very high number of views through some combination of celebrity status and a prolific production ethic.

Table 4: Summary of Machinima by Year

\begin{tabular}{|c|c|c|c|c|}
\hline $\begin{array}{c}\text { Videos } \\
\text { (n=205) }\end{array}$ & $\begin{array}{c}\text { Num. } \\
\text { Videos }\end{array}$ & $\begin{array}{c}\text { Avg. } \\
\text { Views } \\
(\mathrm{k})\end{array}$ & $\begin{array}{c}\text { Sum. } \\
\text { Views } \\
(\mathrm{k})\end{array}$ & $\begin{array}{c}\text { Avg. } \\
\text { Rating } \\
(\%)\end{array}$ \\
\hline 2005 & 11 & 830 & 9,127 & 93.80 \\
\hline 2006 & 47 & 2,425 & 113,997 & 91.35 \\
\hline 2007 & 73 & 1,966 & 143,541 & 88.24 \\
\hline 2008 & 28 & 988 & 33,580 & 93.96 \\
\hline 2009 & 39 & 730 & 28,465 & 94.04 \\
\hline 2010 & 7 & 695 & 4,862 & 96.98 \\
\hline
\end{tabular}

The summary data for authors, videos, views and ratings (Table 2) combined with the individual data for celebrity machinima author "Oxhorn" (Table 3) demonstrate at the level of the author the potential impact of machinima as a form of amateur multimedia. However, what is lost in an emphasis on the author is an understanding of how the broader machinima landscape has shifted over time. Table 4 provides an entry point for a historical analysis by featuring a summary of key metrics for the machinima corpus by year.

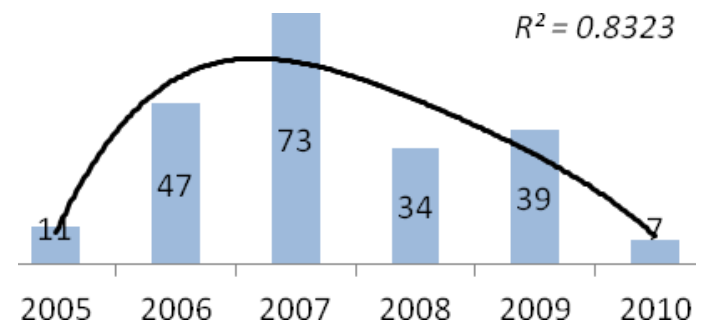

Figure 1: Model of Number of Videos by Year

Figure 1 provides a regression model for the number of videos included in the corpus by year. A single factor analysis of variance (ANOVA) validates the regression model by revealing a significant relation between the number of videos and year of re- 
lease $[f(5,18)=13.092, p<.01]$. Post hoc tests identify that the following pairs are significant at the level of $p<.05:$ 2005-2006, 2005-2007, 2005-2009, 2006-2010, 2007-2008, 2007-2009, 2007-2010, 2008-2010, 2009-2010. Like the model in Figure 1, this data suggests that the number of videos with a significant number of views (>200k) increased from 2005-2007 but then decreased from 2007-2010.

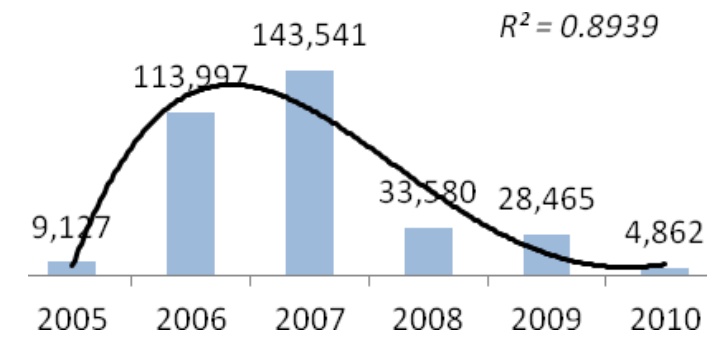

Figure 2: Model of Total Views (k) by Year

In addition to the number of videos per year, the total number of views changes significantly over time. Figure 2 presents a model for the total machinima video views per year in the corpus. A single factor analysis of variance (ANOVA) validates the model by revealing a significant relation across the total number of views per year $[f(5,199)=$ $2.898, p<.02]$. Post hoc tests identify that the following pairs are significant at the level of $p<.05$ : 2005-2006, 2005-2007, 2006-2008, 2006-2009, 2006-2010, 2007-2008, 2007-2009, and 20072010. This data suggests that, along with the number of videos per year, the total number of views for videos in the corpus significantly increases from 2005-2007 and then decreases from 2008-2010.

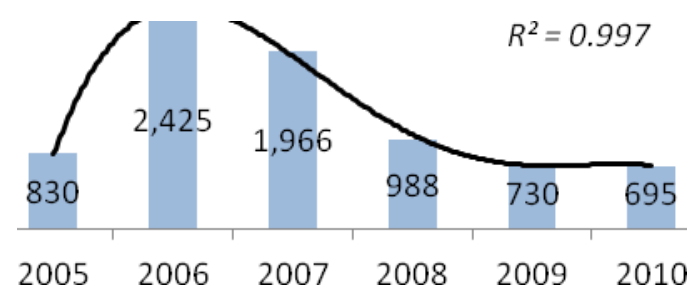

Figure 3: Model of Average Video Views (k) by Year

While the model for total machinima video views by year demonstrates a global trend in views over time, the model of average video views in Figure 3 represents a local view that better displays the effects of breakout videos in any given year. A single factor analysis of variance (ANOVA) revealed a significant relation of average video views over time $[f(5,18)=5.674, p<.01]$. Post hoc tests identify that the following pairs are significant at the level of $\mathrm{p}<$.05: 2005-2006, 2005-2007, 2006-2008, 20062009, 2007-2008, and 2007-2009.

In a manner similar to the data for total video views, this data on average video views further suggests a trend of an increasing number of video views from 2005-2007 with a decrease from 2007-2009. Further, the number of videos and views are not the only significant trends to develop from the analysis of our machinima corpus. The average ratings of videos by year, modelled in Figure 4, reveals a number of significant relations over time.

A single factor analysis of variance (ANOVA) demonstrates a significant relation of average video rating over time $[f(5,198)=2.46, p<.04]$. Post hoc tests identify that the following pairs are significant at the level of $p<.05$ : 2006-2009, 2006-2010, 2007-2008, 2007-2009, and 2007-2010. This data suggests that unlike the number of videos and number of views, the ratings of videos decreased from 2005-2007 and increased from 2007-2010.

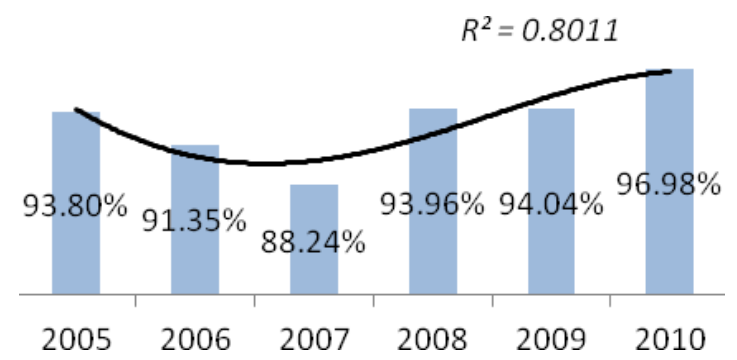

Figure 4: Model of Average Video Ratings (\%) by Year

To better illustrate the trends between videos, views, and ratings over time, we developed the models presented in Figure 5 and Figure 6. Figure 5 maps the model for number of videos in the corpus per year against a model for the total estimated number of new machinima per year. The total estimated number of machinima was derived from YouTube archive searching in one year increments for popular WoW machinima tags/labels and averaging the number of results. While this data is not expected to be accurate in an absolute sense for the number of videos in a year, the estimated machinima numbers do demonstrate a very strong upward trend in total new machinima per year. This significant upward trend sits in opposition to the trend for the number of videos in the corpus which decreases over time.

In Figure 6 the prior models for total video views, average video views, and average video ratings are combined on a standardized scale to visualize the relation between views and ratings. As presented in both Figure 6 and the prior models, view and ratings demonstrate an inverse relationship. While views increase from 2005-2007 and then decline from 2007-2010, ratings do the opposite and decrease from 2005-2007 and increase from 20072010.

\section{DISCUSSION}




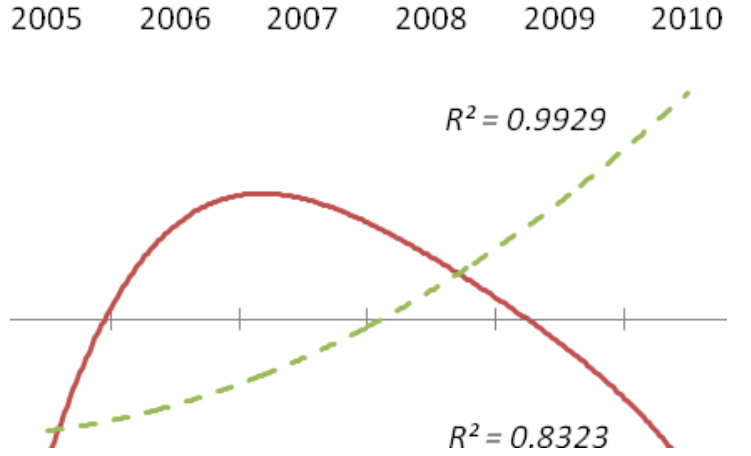

Figure 5: Model of Corpus Video Count (Solid Line) and Total Est. Videos (Dashed Line)

As noted in the Introduction, the project presented in this paper is intended to serve two purposes: (1) to identify a WoW machinima corpus appropriate to study and (2) to test the hypothesis that production techniques, content creation tools, and community standards co-evolved and achieved a point of stability or critical mass. With respect to the first goal, our method of selecting videos based on the number of views was successful in reducing a potential population of videos of over 10,000 to a corpus of 205. However, the means for selecting this corpus raises important questions regarding how well the corpus represents the larger body of machinima.

As Figure 5 shows, the estimated number of new machinima per year increases steadily and dramatically from 2005-2010. However, the number of videos per year represented in our corpus does not increase consistently from 2005-2010. This suggests, in part, that over time the average number of views per video decreases with only an ever smaller percentage of new videos breaking into the elite group of machinima videos with more than 200,000 views. Thus, a machinima corpus identified by views alone results in a model wherein the representative breadth of the corpus declines (compared to the population of videos) while the relative popularity of the videos in the corpus improves over time. High view count videos from the later years may not represent the breadth of machinima available at that time, but they do represent an extremely select group of videos that manage to rise above an ever increasing number of peers-and do so with significantly high user ratings.

However, while a metadata driven corpus offers many benefits (e.g., ready analysability) this type of corpus is not guaranteed to meet all of the broader criteria/goals for a scholarly canon. In particular, the curatorial role of domain experts and critics is suppressed when relying on metadata to form a corpus. While the current corpus successfully represents a populist view of machinima based on measures of audience reception (views and rat-

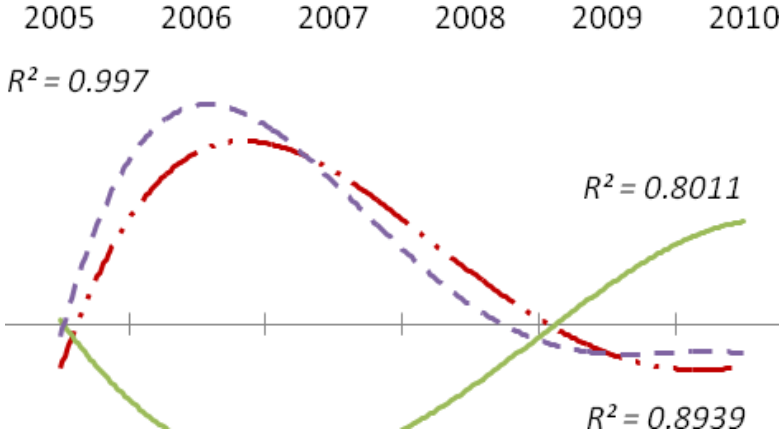

Figure 6: Model of Avg. Views (Dashed Line), Total Views (Dash-Dot Line), and Avg. Ratings (Solid Line) by Year

ings), the corpus does not directly account for the potential influence individual machinima videos have with the creators/producers of WoW machinima. As a result, the opinion of community experts (i.e., popular machinimators, leading machinima bloggers, etc.) is missing from this first pass at a WoW machinima corpus.

Beyond our intention to begin constructing a machinima corpus, this project aimed to provide early evidence of how a large networked creative community might stabilize an emerging digital media aesthetic over time. One of the ways to identify how and when a digital media production oriented community stabilizes is to identify trends in the reception of their product. Figure 6 illustrates such a trend between ratings and views for WoW machinima from 2005-2010.

From Figure 6, we are able to refine our hypothesis concerning the stabilization of an online creative community. Figure 6 demonstrates two major phases in the stabilization of the WoW machinima market. These phases are (a) a period of growth and experimentation marked in years 2005-2007 and (b) a period of relative maturity from 20072010. The 2005-2007 growth phase of WoW machinima is demonstrated by the increasing number of views and decreasing ratings for videos in that time period. These numbers suggest an early period of novelty and experimentation wherein early machinima were capable of achieving great views (thereby inspiring more interest in the media) despite the relatively low quality of the videos themselves.

As an inverse to the growth phase of 2005-2007, the 2007-2010 stage of machinima maturity is marked in Figure 6 by a decreasing view count and increased ratings for machinima in this period. These numbers suggest that within the 2007-2008 year WoW machinima begins to stabilize in terms of community expectations. This maturity phase is perhaps most interesting in the context of the ever increasing number of total machinima videos (i.e., 
Figure 5) available at this time. In this stage, machinima is more available than ever, thereby creating more competition for views. As a result, views per video decline overall but those with high by, the burgeoning WoW community. Several other accomplishments were supports to that primary achievement, including the emergence of community-created machinima production tools (e.g., the

Table 5: Profile of the most viewed machinima in the corpus

A complete chart is available at the following URL: https://sites.google.com/site/wowbcshci11/

\begin{tabular}{|l|c|c|c|c|c|}
\hline \multicolumn{1}{|c|}{ Title } & Date & Author & Views & Rating $(\%)$ & Site \\
\hline Leeroy Jenkins & $8 / 6 / 2006$ & n/a & $21,015,479$ & 96.97 & YouTube \\
\hline World of Warcraft: Dancing & $5 / 8 / 2007$ & animpinabox & $16,786,515$ & 96.42 & YouTube \\
\hline $\begin{array}{l}\text { That's the World of Warcraft } \\
\text { That You Play! }\end{array}$ & $11 / 20 / 2006$ & ibeckman671 & $14,059,141$ & 92.23 & YouTube \\
\hline ROFLMAO! & $1 / 1 / 2007$ & Oxhorn & $12,646,220$ & 91.92 & YouTube \\
\hline 300 World of Warcraft & $3 / 22 / 2007$ & ondskab100 & $8,280,750$ & 88.60 & YouTube \\
\hline
\end{tabular}

enough view counts to make it into the corpus are now doing so with significantly higher viewer ratings. We infer from this that the "right" viewers are increasingly able to find the "right" machinima, which suggests an increasing level of sophistication both among the viewing audience (which is able to find "good" films among the many) and the producer (who is able to target their films at niche audiences for higher ratings).

The data presented here of the increasing size, stabilization, and sophistication of the WoW machinima community serves as preliminary evidence of our hypothesis that bottom-up collective creative practices take a certain amount of time to develop and sustain themselves. Certainly, the development of a thriving aesthetic genre in the form of WoW machinima is a testament to the efforts of machinima producers and consumers to hone their medium. In particular, the data provided in this paper points to the success of the community in appropriating and maximizing the use of a video dissemination network to ensure that good videos (as defined by the community) get to the right audiences.

\section{CONCLUSION}

The structures and practices of online creative communities are of great interest to $\mathrm{HCl}$, one of the key fields responsible for creativity support technologies. Creative networked communities like that of WoW machinima producers manifest their creative practices in often novel ways by leveraging a combination of highly emergent, bottom-up collaboration; networked (and often custom-made) production tools such as the WoW Model Viewer (wowmodelviewer.org), a topic not discussed here, but see (Kow and Nardi, 2010); and a clustering of emergent achievements that are significant and yet not clearly articulated or intended as a long-term goal.

For WoW machinima, we argue the primary achievement was the co-emergence of a new digital media aesthetic that is a fit for, and appreciated
WoW Model Viewer); an expressive visual language that enables video producers to express themselves in a way that resonates with the broader WoW community while relating meaningfully to other major machinima communities (e.g., Halo); a dissemination network that enables video producers and audiences to find each other; and an amateur critical theory of what constitutes "good" WoW machinima shared by the community. In future work, we will study each of these accomplishments. Supporting these sorts of emergent yet valuable creative achievements by loosely organized populations of thousands over a period of years, be they made up of scientists or a video game machinima community, is increasingly part of HCl's creativity support agenda.

\section{ACKNOWLEDGMENTS}

This work was supported by the National Science Foundation (award \#1002772). We are also grateful for the contributions of Natalie DeWitt.

\section{REFERENCES}

Aragon, C., Poon, S., and Silva, C. (2009) The changing face of digital science: new practices in scientific collaborations. In Proc. of ACM CHI 2009. ACM, New York, NY, USA, 4819-4822.

Bardzell, J. (2007) Creativity in Amateur Multimedia: Popular Culture, Critical Theory, and $\mathrm{HCl}$. $\mathrm{Hu}$ man Technology, 3 (1), 12-33.

Dewey, J. (1934) Art as Experience. Perigree. New York, NY, USA.

Kow, Y. M. and Nardi, B. (2010) Culture and Creativity: World of Warcraft Modding in China and the US. In Bainbridge, W.S. (ed.), Online Worlds: Convergence of the Real and the Virtual. Springer, London.

Kriplean, T., Beschastnikh, I., McDonald, D.W., and Golder. S.A. (2007) Community, consensus, coercion, control: $\mathrm{CS}^{*} \mathrm{w}$ or how policy mediates mass participation. In Proc. of ACM GROUP 2007. ACM, New York, NY, USA, 167-176. 
McCarthy, J. and Wright, P. (2004) Technology as Experience. MIT Press, Cambridge, MA, USA.

Pearce, C. (2009) Communities of Play: Emergent Cultures in Multiplayer Games and Virtual Worlds. MIT Press, Cambridge, MA, USA. 